\title{
Three-dimensional osteochondral microtissue to model pathogenesis of osteoarthritis
}

\author{
Thomas P Lozito', Peter G Alexander', Hang Lin', Riccardo Gottardi',2, Anthony Wai-Ming Cheng' and Rocky S Tuan*1
}

\begin{abstract}
Osteoarthritis (OA), the most prevalent form of arthritis, affects up to $15 \%$ of the adult population and is principally characterized by degeneration of the articular cartilage component of the joint, often with accompanying subchondral bone lesions. Understanding the mechanisms underlying the pathogenesis of OA is important for the rational development of disease-modifying OA drugs. While most studies on OA have focused on the investigation of either the cartilage or the bone component of the articular joint, the osteochondral complex represents a more physiologically relevant target because the disease ultimately is a disorder of osteochondral integrity and function. In our current investigation, we are constructing an in vitro three-dimensional microsystem that models the structure and biology of the osteochondral complex of the articular joint. Osteogenic and chondrogenic tissue components are produced using adult human mesenchymal stem cells derived from bone marrow and adipose seeded within biomaterial scaffolds photostereolithographically fabricated with defined internal architecture. A three-dimensionalprinted, perfusion-ready container platform with dimensions to fit into a 96-well culture plate format is designed to house and maintain the osteochondral microsystem that has the following features: an anatomic cartilage/bone biphasic structure with a functional interface; all tissue components derived from a single adult mesenchymal stem cell source to eliminate possible age/tissue-type incompatibility; individual compartments to constitute separate microenvironment for the synovial and osseous components; accessible individual compartments that may be controlled and regulated via the introduction of bioactive agents or candidate effector cells, and tissue/medium sampling and compositional assays; and compatibility with the application of mechanical load and perturbation. The consequences of mechanical injury, exposure to inflammatory cytokines, and compromised bone quality on degenerative changes in the cartilage component are examined in the osteochondral microsystem as a first step towards its eventual application as an improved and high-throughput in vitro model for prediction of efficacy, safety, bioavailability, and toxicology outcomes for candidate disease-modifying OA drugs.
\end{abstract}

Keywords: osteoarthritis, microtissue, osteochondral tissue engineering, mesenchyme stem cells

\section{Osteoarthritis is a disease of the osteochondral junction}

Osteoarthritis (OA) is a chronic degenerative disease of the articular joint that involves cartilage, synovium, ligaments, bone, meniscus, tendon, and periarticular muscle [1]. Cartilage destruction is one of the common characteristics of OA progression and results in malfunction of the affected joint. Normal articular cartilage is comprised of large amounts of extracellular matrix (mainly collagen type II), produced and maintained by chondrocytes, the sole cell type in the cartilage. During

\footnotetext{
*Correspondence: rst13@pitt.edu

${ }^{1}$ Center for Cellular and Molecular Engineering, Department of Orthopaedic

Surgery, 450 Technology Drive, Room 221, Pittsburgh, PA 15219, USA
}

Full list of author information is available at the end of the article disease progression, net loss of cartilage matrix results from an imbalance between cartilage matrix degradation and synthesis by chondrocytes in the cartilage [2-4]. Due to absence of vascularization in the articular cartilage, the capacity for self-repair in cartilage is limited, and currently there is no effective therapy treating OA except for relieving the symptoms of the diseases until the joints need to be replaced by surgery.

OA involves more than simply degeneration of the articular cartilage - OA is in fact a disease of the osteochondral tissue complex. The osteochondral junction is highly structured [5-7]; the uppermost superficial zone is characterized by elongated chondrocytes with collagen fibrils aligning parallel to the articular surface. In the middle/intermediate zone, rounded chondrocytes and collagen fibrils are less organized relative to the surface. In the deep zone, vertical columns of chondrocytes and 
fibers are organized perpendicular to the articular surface. The highest concentration of proteoglycans is found in the deep zone. Adjacent to deep zone cartilage is the calcified cartilage zone, which is characterized by larger and more dispersed hypertrophic chondrocytes. A wavy basophilic matrix, known as the tidemark, highlights the boundary between the deep and calcified cartilage zones. Vertically oriented collagen fibers pass through the tidemark from the deep zone to the calcified cartilage and are important for transferring mechanical forces. Overall, the calcified zone marks the transition from soft cartilage to stiff subchondral bones and is important for attaching the noncalcified cartilage to bone. The subchondral bone is interdigitated with calcified cartilage, but, interestingly, the collagen fibers do not extend from the calcified zone to the bone. This physical linkage between cartilage and bone is a critical component in the pathogenesis of degenerative diseases such as OA.

\section{Pathogenesis of osteoarthritis: bone or cartilage, and bone-cartilage interface}

There exists some debate as to whether OA begins in the cartilage or the bone and whether subchondral bone or articular cartilage is the more appropriate target for disease-modifying OA drug development.

Supporters of the 'bone first' side of the debate maintain that, as the substrate for articular cartilage, subchondral bone plays a support role in cartilage health, and that any perturbations to subchondral bone are amplified as pathological conditions and are transferred from bone to cartilage. For example, studies have shown that osteophyte formation and changes in subchondral bones appear before measurable changes in articular cartilage thickness as well as related joint space narrowing [8-14]. Another group of studies suggest that healthy subchondral bone is essential for healthy cartilage. In tissue plugs cultured in vitro, bone tissue preserves chondrocyte survival [15]. To some extent, the conventional wisdom has been that healthy subchondral bone presents an impenetrable, impermeable barrier. However, growing evidence indicates that cartilage receives nutrients, cytokines, and hormones from bone in vivo, and vice versa [16-18].

Proponents of the 'cartilage first' theory argue that, while early changes to cartilage during OA are clearly coupled to bone alterations via mechanical and soluble factors, changes to the bone seem to be secondary to alterations in articular cartilage [19]. Supporting evidence suggests that OA changes to cartilage alter the mechanical environment of the bone cells and induce them, in turn, to modulate tissue structure. Several studies report that thickening of calcified cartilage along with tidemark advancement contributes to thinning of articular cartilage
[20]. This leads to increased mechanical stresses in the matrix of the deep zone of cartilage and contributes to OA cartilage deterioration [21]. Whatever the side, it is clear that interactions between both bone and cartilage are central to disease progression, and that, in studying $\mathrm{OA}$, bone and cartilage can no longer be considered separately. We thus propose that, in order to appropriately study the osteochondral environment and OA in vitro, three-dimensional microtissue constructs must involve both cartilage and bone.

\section{Design of an adult mesenchymal stem cell-based microsystem of the osteochondral complex}

In view of the findings summarized above, an engineered osteochondral construct that properly incorporates articular cartilage, osteochondral interface (calcified cartilage) and the subchondral bone as an interactive microtissue unit should be of significant utility in elucidating the pathogenesis of degenerative joint diseases as well as assessing the efficacy of potential therapeutics against the disease. Because there are limited differentiated cell sources available for cartilage and bone tissue engineering, adult multipotent mesenchymal stem cells (MSCs), with their well-characterized ability to differentiate into chondrocyte-like cells and osteoblast-like cells, represent a natural candidate cell source for engineering these tissues [22,23].

Human MSCs derived from bone marrow or from adipose are used as the progenitor cell population to engineer the bone, cartilage, and synovium components of the microtissue. However, the microtissue system described here is compatible with constructs derived from any type of progenitor or primary cell. Indeed, induced pluripotent stem cells, with their ability to be propagated to meet the high cell requirements of tissue engineering, represent an attractive, high-quality cell source that we plan to investigate in the future. Regardless of progenitor cells source, a physiologically relevant microsystem of the osteochondral complex must have the following key features: an anatomic biphasic structure with a functional interface; all tissue components derived from a single cell source to eliminate possible age/tissue type incompatibility; individual compartments to constitute separate microenvironment for the synovial and osseous components of the microtissue; accessible individual compartments that may be controlled and regulated via the introduction of bioactive agents or candidate effector cells, and tissue/medium sampling and compositional assays; compatibility with the application of mechanical load and perturbation; and imaging capability to allow for non-invasive functional monitoring.

Here we describe the design for an osteochondral microtissue that is suitable for placement in a 96-well 
tissue culture plate platform (Figure 1A to G). The proposed construct involves a layered osteochondral tissue composite, including (from bottom to top) bone, osteochondral interface, cartilage, and synovium, cultured within a perfusion-ready container mold constructed photolithographically using a biocompatible plastic polymer. The bone construct will be peripherally surrounded by endothelium to simulate the biological effects of blood vessels and the vasculature on OA. Culture-expanded human vascular endothelial cells (ECs) $[24,25]$ are used to form the endothelial lining.

The inclusion of an EC component in an osteochondral tissue construct is a unique feature of our system and is based on the results of numerous studies describing the important role ECs play in regulating bone biology [26]. ECs release factors such as fibroblast growth factors, IL-1, and IL-6, and nitric oxide that influence both bone and osteoclast behavior, thereby regulating bone formation and resorption. In particular, ECs provide a robust source of bone morphogenetic protein-2 that enhances the osteogenic phenotype in bone and boneprogenitor cells $[27,28]$. In turn, ECs are the target of many bone-derived signals, such as parathyroid hormone, insulin-like growth factors types 1 and 2, basic fibroblast growth factor, platelet-derived growth factor, and vascular endothelial growth factor. To demonstrate the effects of this crosstalk between ECs and bone cells in our system, we conducted preliminary studies in which native bone plugs were cultured with collagen gels seeded with or without ECs and cultured for 4 weeks. Samples of bone coated with collagen gels containing ECs produce more new bone matrix and osteoprotegerin, indicating activation of anabolic bone pathways (Figure $1 \mathrm{H}$ to I). Future work will be aimed at assessing whether crosstalk with ECs mitigates the negative effects of injurious mechanical and chemical stresses on bone behavior.

Each tissue type is formulated with the use of projection stereolithography to incorporate internal threedimensional spatial features that will permit optimal tissue formation and medium perfusion [29]. For example, channels $500 \mu \mathrm{m}$ in diameter can be fabricated within the bone element to aid in nutrient dispersion throughout the construct. Bone is formed by seeding and culturing MSCs in photocrosslinked collagen/hydroxyapatite [30] (Figure 2). Collagen and hydroxyapatite, or $\mathrm{Ca}_{10}\left(\mathrm{PO}_{4}\right)_{6}(\mathrm{OH})_{2}$, are primary components of bone, and both are frequently used in tissue engineered bone constructs. Cartilage is engineered by seeding MSCs in photo-activated/crosslinked collagen/chitosan gel and treated with transforming growth factor- $\beta 3$ [31]. Chitosan was chosen because it shares some structural characteristics with glycosaminoglycans, a major matrix component of cartilage responsible for many of its unique mechanical properties [32]. With its many primary amine groups, chitosan also aids in collagen crosslinking [33]. The osteochondral interface is formed by placing a layer of MSC-laden collagen type I hydrogel between the chondral and osseous layers [34]. The synovial lining is generated with MSCs seeded in crosslinked polyethylene glycol alone and cultured in non-inductive medium. These conditions were shown in preliminary experiments to be capable of maintaining a fibroblastic phenotype in MSCs. As previously mentioned, the endothelial component consists of ECs embedded in collagen to surround the osteochondral elements. Collagen gels were chosen for their susceptibility to modification and contraction by ECs and osteoblasts, resulting in a tight fit around the osteoblast construct.

This osteochondral microtissue design specifies two separate circulating feeding/delivery systems, which may be mixed if desired. One system supplies the upper synovial compartment and is separated by a rigid wall with $20 \mu \mathrm{m}$ pores with or without a $0.2 \mu \mathrm{m}$ filter lining. The outer wall is layered with ECs that adhere and develop after the cells are delivered by perfusion once the construct is assembled. The inner layer is lined with a collar of MSC-embedded photo-polymerized hydrogel to constitute the synovium. The second system supplies the bony tissue component and is separated from the bone with a rigid wall with $\geq 20 \mu \mathrm{m}$ pores, thus delivering nutrients as well as allowing ECs and other cells to adhere and migrate into the bony tissue and create new biologically relevant niches.

Towards fully simulating the osteochondral microenvironment, the proposed microtissue system will include mechanical loading modalities. The initial design of the loading device involves a $3 \mathrm{~mm}$ loading surface with nonloading position $<0.5 \mathrm{~mm}$ from the cartilage surface and loading of $5 \%$ strain $(100 \mu \mathrm{m})$ at $0.1 \mathrm{~Hz}$. Reports in the literature suggest that this combination of strain and loading rate should be chondro-stimulatory in engineered cartilage constructs [35-37]. Furthermore, extreme loading can be applied in conjunction with stimulation by biochemical stresses to simulate physical injury within the microtissue system (Figure 2).

The most attractive aspect of the microtissue described here will be its ability to mimic the tissue relationships within the osteochondral complex of the articular joint and to characterize responses to mechanical, pathological, and inflammatory insults. The application of the system toward these types of studies will depend on first validating its behavior under nonstressed conditions to confirm proper matrix production, differentiation marker expression, and tidemark development. Next the system will be perturbed with mechanical and chemical stress to demonstrate that the microtissue responds according to published in vivo studies. Once validated, the system can be used to investigate biological processes not easily 

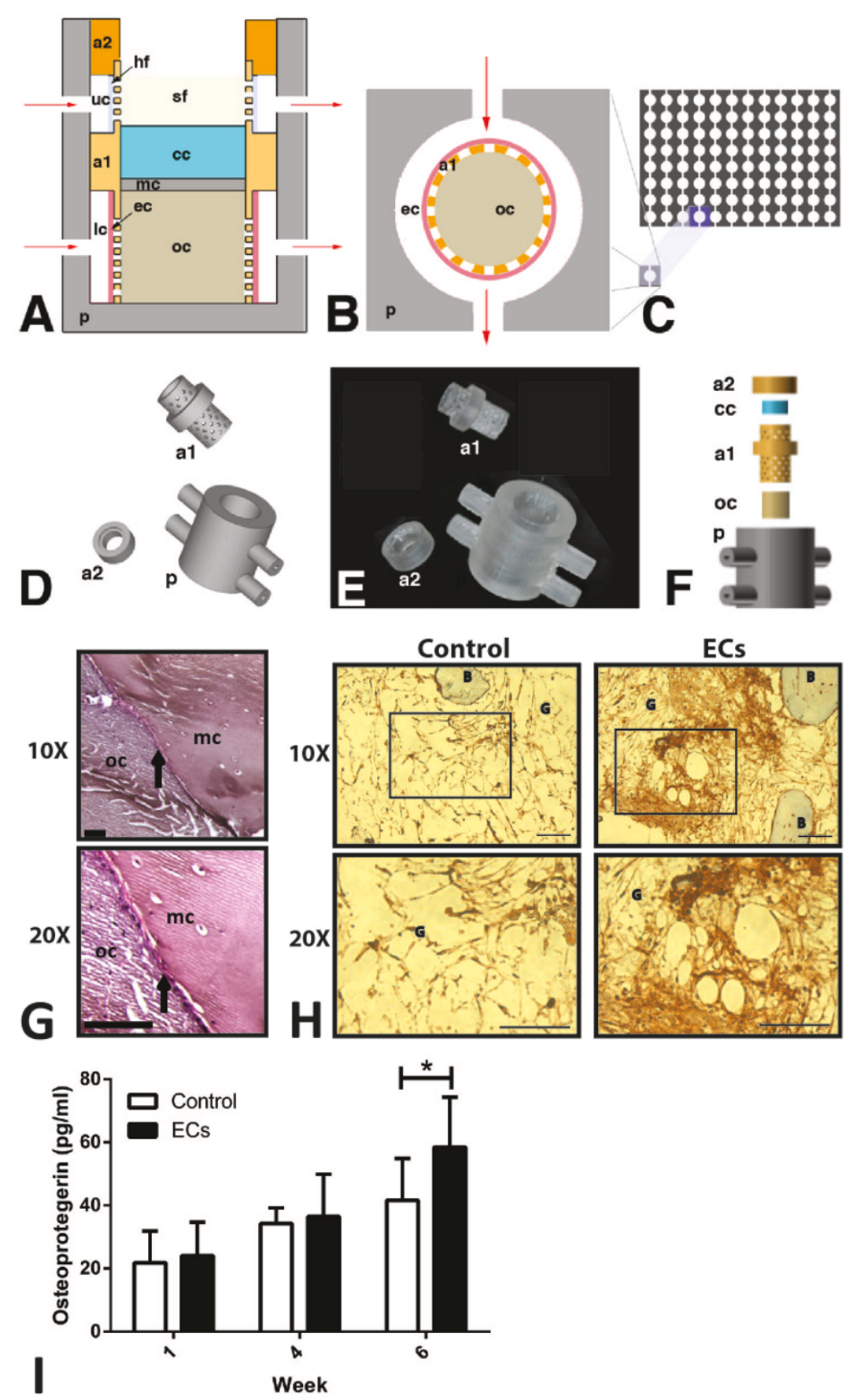

Figure 1. Osteochondral microtissue design, fabrication, and validation. (A) Schematic diagram of one microtissue assembly within a single well platform, cross-sectional side view. cc, chondrocyte construct; ec, endothelial construct; hf, human fibroblasts; lc, lower medium conduit; mc, mesenchymal construct; oc, osteoblast construct; $\mathrm{p}$, platform; sf, synovial fluid; uc, upper medium conduit; a1, lower adapter with porous screen to allow communication between the Ic and oc and the uc and cc chamber; a2, upper adapter, to seal the upper conduit. Red arrows indicate direction of fluid flow. (B) Schematic of one microtissue assembly within a single well platform showing fluid flow (red arrows) of the Ic and its relation to the endothelial layer (ec), oc and the porous barrier (a1). (C) Combination of multiple microtissue platform configurations, 96-well format, and single well format. (D) Three-dimensional model fabricated microtissue components. (E) Microtissue components fabricated with E-shell 300 ${ }^{\text {TM }}$ (EnvisionTec, Gladbeck, Germany) polymer resin using projection stereolithography. (F) Order of microtissue assembly before culture. (G) Histological view of interface between osteoblast and mesenchymal construct on a fully assembled microtissue construct after 6 weeks of culture. Arrows indicate a dense structure between the two layers. Bar $=100 \mu \mathrm{m}$. (H) Native bone plugs in collagen gel with or without endothelial cells (ECS) cultured for 4 weeks immunohistochemically stained for osteoprotegerin. B, bone plug; G, collagen gel. Bar = $100 \mu \mathrm{m}$. (I) Osteoprotegerin ELISA analysis of media samples conditioned by bone plugs coated in collagen gel with or without ECs cultured for 1, 4, or 6 weeks. ${ }^{*} P=0.0362$. 


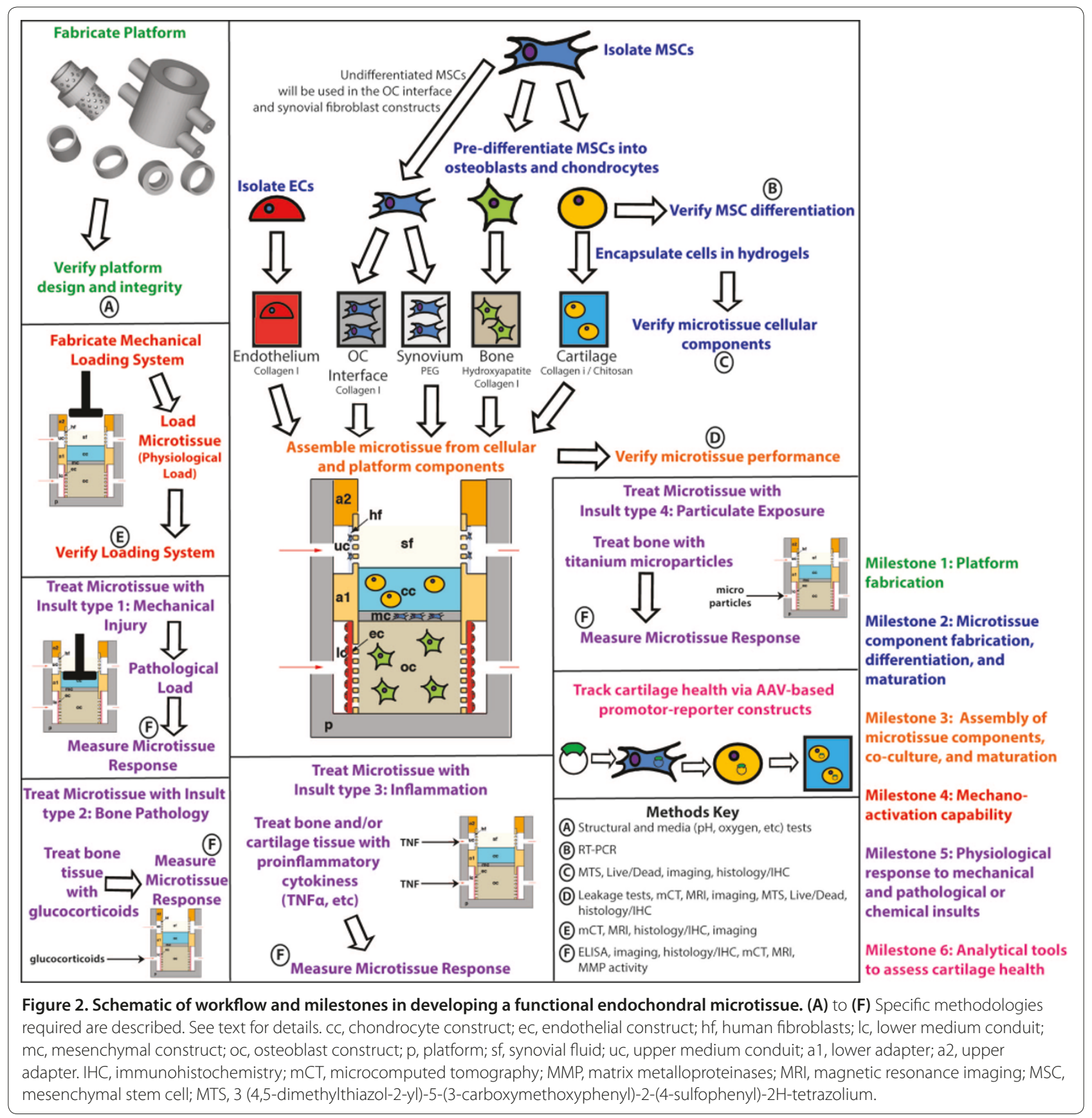

studied by traditional means. For example, to study the effects of mechanical injury, the cartilage component can be pre-injured prior to microtissue assembly to study the effects of damaged cartilage on bone health. Alternatively, the assembled and matured microtissue can be impacted in situ to study changes in cartilage and bone anabolic/ catabolic pathways and disruption of the tidemark. Similarly, the microtissue system can be employed as a high-throughput in vitro model to assess the effects of treatment with glucocorticoids, proinflammatory cytokines, anti-inflammatory biologics, and even titanium microparticles on osteochondral health (Figure 2). The microtissue system thus offers novel capabilities for investigating the pathogenic mechanisms of $\mathrm{OA}$ as well as serving as a high-throughput platform to test candidate disease-modifying OA drugs.

\section{Abbreviations}

EC, endothelial cell; ELISA, enzyme-linked immunosorbent assay; IL, interleukin; MSC, mesenchymal stem cell; OA, osteoarthritis.

\section{Competing interests}

The authors declare that they have no competing interests. 


\section{Acknowledgements}

This work was supported by grants from the Commonwealth of Pennsylvania Department of Health, the National Institutes of Health (1U18 TR000532-01), and the Ri.MED Foundation. The publication costs for this article were funded by National Institutes of Health grant U18TR000532.

\section{Declarations}

Publication of this supplement has not been supported by sponsorship. Articles have undergone the journal's standard review process. The Editors declare that they have no competing interests.

This article has been published as part of Stem Cell Research \& Therapy Volume 4 Supplement 1, 2013: Stem cells on bioengineered microphysiological platforms for disease modeling and drug testing. The full contents of the supplement are available online at http://www.stemcellres.com/supplements/4/S1.

\section{Author details}

${ }^{1}$ Center for Cellular and Molecular Engineering, Department of Orthopaedic Surgery, 450 Technology Drive, Room 221, Pittsburgh, PA 15219, USA. ${ }^{2}$ Ri. MED Foundation, via Bandiera 11-90133 Palermo, Italy.

Published: 20 December 2013

\section{References}

1. Hunter DJ, Felson DT: Osteoarthritis. BMJ 2006, 332:639-642

2. Mueller MB, Tuan RS: Anabolic/catabolic balance in pathogenesis of osteoarthritis: identifying molecular targets. PM R 2011, 3:S3-S11.

3. Fukui N, Purple CR, Sandell LJ: Cell biology of osteoarthritis: the chondrocyte's response to injury. Curr Rheumatol Rep 2001, 3:496-505.

4. Krasnokutsky S, Attur M, Palmer G, Samuels J, Abramson SB: Current concepts in the pathogenesis of osteoarthritis. Osteoarthritis Cartilage 2008, 16(Suppl 3):S1-S3.

5. Yang PJ, Temenoff JS: Engineering orthopedic tissue interfaces. Tissue Eng Part B Rev 2009, 15:127-141.

6. Keeney M, Pandit A: The osteochondral junction and its repair via bi-phasic tissue engineering scaffolds. Tissue Eng Part B Rev 2009, 15:55-73.

7. Grogan SP, Miyaki S, Asahara H, D'Lima DD, Lotz MK: Mesenchymal progenitor cell markers in human articular cartilage: normal distribution and changes in osteoarthritis. Arthritis Res Ther 2009, 11:R85.

8. Buckland-Wright C: Subchondral bone changes in hand and knee osteoarthritis detected by radiography. Osteoarthritis Cartilage 2004, 12(Suppl A):S10-S19.

9. Roos EM: Joint injury causes knee osteoarthritis in young adults. Curr Opin Rheumatol 2005, 17:195-200.

10. Muraoka T, Hagino H, Okano T, Enokida M, Teshima R: Role of subchondral bone in osteoarthritis development: a comparative study of two strains of guinea pigs with and without spontaneously occurring osteoarthritis. Arthritis Rheum 2007, 56:3366-3374.

11. Hayami T, Pickarski M, Zhuo Y, Wesolowski GA, Rodan GA, Duong le T: Characterization of articular cartilage and subchondral bone changes in the rat anterior cruciate ligament transection and meniscectomized models of osteoarthritis. Bone 2006, 38:234-243.

12. Sugano N, Noble PC, Kamaric E, Salama JK, Ochi T, Tullos HS: The morphology of the femur in developmental dysplasia of the hip. J Bone Joint Surg Br 1998, 80:711-719.

13. Laborie LB, Lehmann TG, Engesaeter IO, Eastwood DM, Engesaeter LB, Rosendahl K: Prevalence of radiographic findings thought to be associated with femoroacetabular impingement in a population-based cohort of 2081 healthy young adults. Radiology 2011, 260:494-502.

14. Steppacher SD, Tannast M, Ganz R, Siebenrock KA: Mean 20-year followup of Bernese periacetabular osteotomy. Clin Orthop Relat Res 2008, 466:1633-1644.

15. Amin AK, Huntley JS, Simpson AH, Hall AC: Chondrocyte survival in articular cartilage: the influence of subchondral bone in a bovine model. J Bone Joint Surg Br 2009, 91:691-699.

16. Pan J, Zhou X, Li W, Novotny JE, Doty SB, Wang L: In situ measurement of transport between subchondral bone and articular cartilage. J Orthop Res 2009, 27:1347-1352.
17. Imhof H, Breitenseher M, Kainberger F, Trattnig S: Degenerative joint disease: cartilage or vascular disease? Skeletal Radiol 1997, 26:398-403.

18. Imhof H, Sulzbacher I, Grampp S, Czerny C, Youssefzadeh S, Kainberger F: Subchondral bone and cartilage disease: a rediscovered functional unit. Invest Radio/ 2000, 35:581-588.

19. Heinegard D, Saxne T: The role of the cartilage matrix in osteoarthritis. Nat Rev Rheumatol 2011, 7:50-56.

20. Goldring MB, Goldring SR: Articular cartilage and subchondral bone in the pathogenesis of osteoarthritis. Ann N Y Acad Sci 2010, 1192:230-237.

21. Burr DB: Anatomy and physiology of the mineralized tissues: role in the pathogenesis of osteoarthrosis. Osteoarthritis Cartilage 2004, 12(Suppl A):S20-S30.

22. Chen FH, Rousche KT, Tuan RS: Technology insight: adult stem cells in cartilage regeneration and tissue engineering. Nat Clin Pract Rheumatol 2006, 2:373-382.

23. Kraus $\mathrm{KH}$, Kirker-Head C: Mesenchymal stem cells and bone regeneration. Vet Surg 2006, 35:232-242.

24. van Beijnum JR, Rousch M, Castermans K, van der Linden E, Griffioen AW Isolation of endothelial cells from fresh tissues. Nat Protoc 2008, 3:1085-1091.

25. Kriehuber E, Breiteneder-Geleff S, Groeger M, Soleiman A, Schoppmann SF, Stingl G, Kerjaschki D, Maurer D: Isolation and characterization of dermal lymphatic and blood endothelial cells reveal stable and functionally specialized cell lineages. J Exp Med 2001, 194:797-808.

26. Collin-Osdoby P: Role of vascular endothelial cells in bone biology. J Cell Biochem 1994, 55:304-309.

27. Kaigler D, Krebsbach PH, West ER, Horger K, Huang YC, Mooney DJ: Endothelial cell modulation of bone marrow stromal cell osteogenic potential. FASEB J 2005, 19:665-667.

28. Bouletreau PJ, Warren SM, Spector JA, Peled ZM, Gerrets RP, Greenwald JA, Longaker MT: Hypoxia and VEGF up-regulate BMP-2 mRNA and protein expression in microvascular endothelial cells: implications for fracture healing. Plastic Reconstr Surg 2002, 109:2384-2397.

29. Lin H, Zhang D, Alexander PG, Yang G, Tan J, Cheng AW, Tuan RS: Application of visible light-based projection stereolithography for live cell-scaffold fabrication with designed architecture. Biomaterials 2013, 34:331-339.

30. Hillig WB, Choi Y, Murthy S, Natravali N, Ajayan P: An open-pored gelatin/ hydroxyapatite composite as a potential bone substitute. J Mater Sci Mater Med 2008, 19:11-17.

31. Yan LP, Wang YJ, Ren L, Wu G, Caridade SG, Fan JB, Wang LY, Ji PH, Oliveira JM, Oliveira JT, Mano JF, Reis RL: Genipin-cross-linked collagen/chitosan biomimetic scaffolds for articular cartilage tissue engineering applications. J Biomed Mater Res A 2010, 95:465-475.

32. Di Martino A, Sittinger M, Risbud MV: Chitosan: a versatile biopolymer for orthopaedic tissue-engineering. Biomaterials 2005, 26:5983-5990.

33. Ma L, Gao C, Mao Z, Zhou J, Shen J, Hu X, Han C: Collagen/chitosan porous scaffolds with improved biostability for skin tissue engineering. Biomaterials 2003, 24:4833-4841.

34. Cheng HW, Luk KD, Cheung KM, Chan BP: In vitro generation of an osteochondral interface from mesenchymal stem cell-collagen microspheres. Biomaterials 2011, 32:1526-1535.

35. Elder SH, Goldstein SA, Kimura JH, Soslowsky LJ, Spengler DM: Chondrocyte differentiation is modulated by frequency and duration of cyclic compressive loading. Ann Biomed Eng 2001, 29:476-482.

36. McMahon LA, Reid AJ, Campbell VA, Prendergast PJ: Regulatory effects of mechanical strain on the chondrogenic differentiation of MSCs in a collagen-GAG scaffold: experimental and computational analysis. Ann Biomed Eng 2008, 36:185-194.

37. Huang AH, Farrell MJ, Kim M, Mauck RL: Long-term dynamic loading improves the mechanical properties of chondrogenic mesenchymal stem cell-laden hydrogel. Eur Cells Mater 2010, 19:72-85.

\section{doi:10.1186/scrt367}

Cite this article as: Lozito TP, et al:: Three-dimensional osteochondral microtissue to model pathogenesis of osteoarthritis. Stem Cell Research \&

Therapy 2013, 4(Suppl 1):S6. 